\title{
Posttraumatic growth (PTG) and posttraumatic depreciation (PTD) across ten countries: Global validation of the PTG-PTD theoretical model ${ }^{\text {is }}$
}

Kanako Taku ${ }^{\mathrm{a}, *}$, Richard G. Tedeschi ${ }^{\mathrm{b}}$, Jane Shakespeare-Finch ${ }^{\mathrm{c}}$, Daniel Krosch ${ }^{\mathrm{c}}$, Georgina David ${ }^{\mathrm{c}}$, Doris Kehl ${ }^{\mathrm{d}}$, Selina Grunwald ${ }^{\mathrm{d}}$, Annunziata Romeo ${ }^{\mathrm{e}}$, Marialaura Di Tella ${ }^{\mathrm{e}}$, Kiyoko Kamibeppu ${ }^{\mathrm{f}}$, Takafumi Soejima ${ }^{\mathrm{f}}$, Kohichi Hiraki ${ }^{\mathrm{g}}$, Rebekah Volgin ${ }^{\mathrm{c}}$, Sandesh Dhakal ${ }^{\mathrm{h}}$, Mariusz Zięba ${ }^{\mathrm{i}}$, Catarina Ramos ${ }^{\mathrm{j}, 1}$, Romina Nunes ${ }^{\mathrm{k}}$, Isabel Leal ${ }^{1}$, Patrícia Gouveia ${ }^{j}$, Carolina C. Silva ${ }^{1, m}$, Pamela Núñez Del Prado Chaves ${ }^{\mathrm{n}}$, Claudia Zavala ${ }^{\mathrm{n}}$, Andrea Paz ${ }^{\mathrm{o}}$, Emre Senol-Durak $^{\mathrm{p}}$, Atsushi Oshio ${ }^{\mathrm{q}}$, Amy Canevello ${ }^{\mathrm{b}}$, Arnie Cann ${ }^{\mathrm{b}}$, Lawrence G. Calhoun ${ }^{\mathrm{b}}$

${ }^{a}$ Department of Psychology, Oakland University, Rochester, MI, USA

${ }^{\mathrm{b}}$ Department of Psychological Science, UNC Charlotte, Charlotte, NC, USA

${ }^{\mathrm{c}}$ School of Psychology and Counselling, Faculty of Health, Queensland University of Technology, Australia

${ }^{\mathrm{d}}$ Institute for Medical Psychology, University Medicine Greifswald, Greifswald, Germany

${ }^{\mathrm{e}}$ Department of Psychology, University of Turin, Italy

${ }^{\mathrm{f}}$ Department of Family Nursing, Division of Health Sciences and Nursing, Graduate School of Medicine, The University of Tokyo, Tokyo, Japan

${ }^{g}$ Nagasaki Wesleyan University, Nagasaki, Japan

${ }^{\mathrm{h}}$ Central Department of Psychology, Tribhuvan University, Kathmandu, Nepal

${ }^{\text {i }}$ SWPS University of Social Sciences and Humanities, Centre for Trauma, Crisis and Growth, Poznan, Poland

${ }^{\mathrm{j}}$ LabPSI - Laboratório de Psicologia, CiiEM - Centro de Investigação Interdisciplinar Egas Moniz, IUEM - Instituto Universitário Egas Moniz, Caparica, Portugal

${ }^{\mathrm{k}}$ ISPA - Instituto Universitário, Lisbon, Portugal

${ }^{1}$ WJCR - William James Center for Research, ISPA - Instituto Universitário, Lisbon, Portugal

${ }^{\mathrm{m}}$ ADAPT Centre, School of Nursing and Midwifery, Trinity College Dublin, Dublin, Ireland

${ }^{n}$ Pontificia Universidad Catolica del, Peru

${ }^{\circ}$ Universidad Peruana Cayetano Heredia, Peru

${ }^{\mathrm{P}}$ Department of Psychology, Bolu Abant Izzet Baysal University, Bolu, Turkey

${ }^{\mathrm{q}}$ Faculty of Letters, Arts and Sciences, Waseda University, Tokyo, Japan

A R T I C L E I N F O

\section{Keywords:}

Posttraumatic growth

Posttraumatic depreciation

Culture

Core beliefs

Event centrality

Rumination

Disclosure

\begin{abstract}
A B S T R A C T
This study examined the relationships between posttraumatic growth (PTG) and posttraumatic depreciation (PTD) across 10 countries and assessed the factorial invariance of the standardized inventory assessing PTG and PTD, the PTGDI-X, the expansion of the PTGI-X (Tedeschi et al., 2017). We also investigated the roles of social and cognitive factors in PTG and PTD. Data were collected from participants who identified that their most stressful life experience met the definition of trauma in Australia, Germany, Italy, Japan, Nepal, Peru, Poland, Portugal, Turkey, and the US. The participants completed the PTGDI-X and inventories measuring posttraumatic stress disorder symptoms, and predictor variables such as reexamination of core beliefs, centrality of the event, rumination, and disclosures. Results identified universal aspects such as equivalence of factor loadings of the PTGDI-X and the impact of positive/negative disclosure on PTG and PTD. Results also revealed culture-specific aspects, including the relationships between PTG and PTD, and different patterns of cognitive predictors for PTG and PTD. The current study offered the insight that, for the first time using international data, positive and negative post-trauma changes are not likely to be on opposing ends of one dimension, and it is essential to use the PTGDI-X to better understand both positive and negative aspects of post-traumatic experiences.
\end{abstract}

\footnotetext{
A part of this study was funded by the grant 2013/10/E/HS6/00502 from the National Science Center, Poland, awarded to Mariusz Zięba.

All authors declare that the research was conducted in the absence of any commercial or financial relationships that could be construed as a potential conflict of interest.

* Corresponding author at: Department of Psychology, Oakland University, 654 Pioneer Drive, 123 Pryale Hall, Rochester, MI 48309, USA.

E-mail address: taku@oakland.edu (K. Taku).
} 


\section{Introduction}

Posttraumatic growth (PTG) or positive psychological changes have been reported by people around the world who struggled with the aftermath of a highly stressful and potentially traumatic life event (Weiss \& Berger, 2010). The phenomenon of PTG are generally observed in the five domains (Shakespeare-Finch et al., 2013). The first domain is an increased sense of self-reliance or strength. The second lies in the changed quality of relationship that includes more compassion and feeling of connectedness. The third domain reflects the experience of finding a new or different path in life that would not have been a part of one's life if there had been no adversity. The fourth domain includes a greater appreciation for life. And the fifth domain contains spiritual and existential changes that may include a deeper understanding of philosophical questions. These five domains have been validated in quantitative studies (Taku et al., 2008) and identified by thematic analyses (Mosher et al., 2017).

While studies have examined the nature of these five domains of PTG, researchers have pointed out that people experience both positive and negative changes in the aftermath of a trauma (Cann, Calhoun, Tedeschi, \& Solomon, 2010). For example, people who became more self-reliant might also recognize how much they need others' support and felt more vulnerable than they thought they were. People who learned how wonderful and helpful other people are might also have learned how cruel and inconsiderate some people could be. These seemingly opposite changes were referred to as posttraumatic depreciation (PTD) and have been recognized in the same five domains (Baker et al., 2008; Cann et al., 2010). A concept of PTD reflects the opposite side of PTG to more comprehensively understand both positive and negative changes that may happen after a trauma in a parallel way. Studies indicate that some people experience both PTG and PTD concurrently. A longitudinal study with tsunami survivors, for example, revealed that people who experienced multiple trauma showed a greater level of both PTG and PTD than those who experienced a single exposure (Michélsen et al., 2017). In addition, PTG is more reported than PTD (Cann, Calhoun, Tedeschi, Kilmer, et al., 2010; Michélsen et al., 2017). However, little research has been conducted to examine the domain level of PTD.

Several studies have also attempted to capture PTG and the corresponding negative changes by applying a single dimension ranging from "much worse or less than before" to "much better or more than before", while placing no change or "same as before" in the middle, assuming that people should experience either positive or negative changes as a result of a trauma (e.g., Nordstrand et al., 2017). However, it is important to allow study participants to report positive and negative changes that may happen concurrently, because studies that examined PTG and PTD generally support the independent nature of these two constructs (Barrington \& Shakespeare-Finch, 2013). One study demonstrated an inverted-U shape relationship between PTG and PTD, suggesting that PTG is greatest when the level of PTD is not too low or high (Michélsen et al., 2017). However, this finding has not been replicated. The first aim of the present study was to investigate the relationship of PTG and PTD, as well as posttraumatic stress disorder (PTSD) symptoms across countries, as a meta-analytical study has suggested that PTG is also correlated with PTSD symptoms in a positive or curvilinear manner (Shakespeare-Finch \& Lurie-Beck, 2014). We also assess the factorial invariance of the standardized inventeory assessing PTG and PTD, the Posttraumatic Growth and Posttraumatic Depreciation Inventory - Expanded version (PTGDI-X) and provide the psychometric properties.

The second aim was to identify the psycho-social factors that would explain PTG and PTD. A theoretical model depicting the PTG process has been refined across diverse samples and cultural contexts (Tedeschi et al., 2018, for review). According to the model, three components primarily explain PTG and possibly PTD. First, a potentially traumatic life event may challenge one's core beliefs, a broad set of fundamental beliefs about self, others, and the world (Cann, Calhoun, Tedeschi, Kilmer, et al., 2010). When the event challenges one's core beliefs, individuals are forced to rebuild their cognitive system. The degree of challenged core beliefs has been shown to be one strong predictor for PTG (Wilson et al., 2014), however, its relationship with PTD is unknown. Similar to this concept, event centrality, the extent to which the memory of adversity becomes central to one's identity (Berntsen \& Rubin, 2006), has been suggested to predict both PTG and PTD (Allbaugh et al., 2016). We therefore hypothesized that across the cultures, challenged core beliefs would be positively associated with PTG, whereas event centrality would be positively associated with both PTG and PTD because the event may become central to one's identity whether it leads to either positive or negative change after a trauma (Groleau et al., 2013).

Second, cognitive processing followed by challenged core beliefs also plays a major role in PTG (Cann et al., 2011). Two distinct types have been identified. "Intrusive rumination" often occurs as an automatic uncontrollable negative thought while "deliberate rumination" is more effortful, constructive, and intentional in nature. Positive impact of deliberate rumination on PTG has been consistently reported (e.g., Tedeshci \& Blevins, 2015), whereas the impact of intrusive rumination on PTG is equivocal, but mostly weak or none (Gul \& Karanci, 2017). On the other hand, intrusive rumination showed a positive association with PTD (Cann, Calhoun, Tedeschi, Kilmer, et al., 2010) and deliberate rumination showed a smaller, although still positive, relationship with PTD (Allbaugh et al., 2016). We hypothesized that intrusive rumination would be associated with PTD, whereas deliberate rumination would be associated with PTG.

The third component that explains PTG and PTD are the impact of disclosure (Tedeschi et al., 2018). Self-disclosure has been shown to foster PTG regardless of different methods of disclosure (Slavin-Spenny et al., 2011). However, disclosure may lead to PTD when the person perceives his/her disclosure was not well-received by others, because it would lead to a sense of regret and lack of emotional bonds. If, however, the person recognized that his/her disclosure was well-received by others, it would lead to PTG. Little research was done about the impact of positive and negative experiences of self-disclosure on PTG and PTD.

In summary, we investigated the potentially different patterns of these theory-driven predictors, that is, core belief disruption (Cann, Calhoun, Tedeschi, Kilmer, et al., 2010) and event centrality (Berntsen \& Rubin, 2006), cognitive processing (Cann et al., 2011), and positive and negative perceptions about self-disclosure (Müller et al., 2000) on PTG and PTD.

\section{Method}

\subsection{Participants and procedure}

The participants responded to demographic measures, reported one major traumatic life experience, and indicated whether their event met the definition of a trauma according to DSM-IV (American Psychiatric Association, 2000). Those who met the criteria then rated the severity of the event, stressfulness when the event happened, the stressfulness when they took the survey, and the level of resolution. They then responded to a series of inventories that measure PTG, PTD, and the relevant social cognitive factors based on their traumatic experiences. Sample information is presented in Supplemental Table S1.

\subsubsection{Australian sample}

Of 231 adults who participated (189 university students and 42 members of community sample), 226 identified that their experience met the definition of a trauma $(18.58 \%$ women; 18 to 69 years old; Mage $=26.28$; $S D=12.14$ ). Data were collected online. University students were offered course credit, and the members from the community participated with no incentives. The research was approved by 
the Queensland University of Technology Human Research Ethics Committee.

\subsubsection{German sample}

A total of 202 adults who met the criteria participated in an online survey. The participants, an adult community sample, included emergency personnel working in the field of civil protection and trauma survivors from the general population $(67.82 \%$ women; 18 to 78 years old; Mage $=34.71 ; \mathrm{SD}=12.01$ ). Participants could win one of twenty 15 euros Amazon vouchers in a voluntary raffle. The research was approved by the Ethics Committee of the University Medicine Greifswald, Germany.

\subsubsection{Italian sample}

Initially, students of the Department of Psychology at the [removed for blind review] were involved in the recruitment of potential participants. The volunteers were contacted by e-mail and informed about the current research study. A total of 321 participants who met the inclusion criteria, agreed to take part in the study, and completed the online survey $(76.01 \%$ women; 18 to 72 years old; Mage $=29.98$; $\mathrm{SD}=11.34$ ). The study was approved by the bioethics committee of the University of Turin, Italy.

\subsubsection{Japanese sample}

Of 514 adults, including undergraduate students and firefighters, who participated in the survey, 130 met the criteria for traumatic event (54.62\% women; 19 to 64 years old; Mage $=25.75$; SD $=10.76$ ). Participants were recruited in a fire station and from psychology classes at seven universities. Participants did not receive any compensation or course credit for participation. The survey was conducted using a paper and pencil survey in a group setting. The study was approved by the Institutional Review Board of Tokyo University, Japan.

\subsubsection{Nepali sample}

Of 247 adults, 196 met inclusion criteria and were instructed to respond to the survey on the basis of the most traumatic event they have experienced, including the earthquake that happened in 2015 ( $65.82 \%$ women; 18 to 83 years old; Mage $=24.45$; $S D=8.39$ ). It was a paper pencil survey and the participants did not receive any compensation for participating in the survey.The study was approved by Government of Nepal, Nepal Health Research Council.

\subsubsection{Peruvian sample}

A total of 201 adults who met the inclusion criteria participated in an online survey $(75.62 \%$ women; 18 to 61 years old; Mage $=25.29$; $\mathrm{SD}=9.63$ ). They were recruited in different institutions such as the national oncological hospital and the children's hospital. They were instructed to identify one traumatic event that happened within the past two years. The data was part of a bigger research project that has IRB approval from Instituto Nacional de Enfermedades Neoplasicas (INEN, the National Oncological Hospital in Peru) and Pontificia Universidad Catolica del Peru.

\subsubsection{Polish sample}

A total of 287 undergraduate and graduate students who met the criteria participated in the study (85.02\% women; 18 to 67 years old; Mage $=28.24 ; \quad S D=8.99$ ). They were recruited from psychology classes at three campuses of a university. Participants received credits for their participation. The study was approved by the SWPS University of Social Sciences and Humanities Institutional Research Ethics Body.

\subsubsection{Portuguese sample}

A total of 181 adults met the criteria and participated in the study $(73.48 \%$ women; 18 to 73 years old; Mage $=32.34$; $\mathrm{SD}=12.91)$. They were recruited from two universities as well as from the community. In both samples the survey was conducted by personal face-to-face contact and via the dissemination of an online survey link through email and social networks. The participants did not receive any compensation or course credits for their participation. The research was approved by the ISPA-Instituto Universitário and by the Instituto Universitário Egas Moniz, Portugal.

\subsubsection{Turkish sample}

A total of 169 adults were recruited from introductory psychology classes at one university as well as the community $(78.11 \%$ women; 18 to 83 years old; Mage $=24.45$; $S D=8.39$ ). The online survey link was shared with students at classes and website of a Turkish researcher to recruit potential participants who had a traumatic experience. All participants voluntarily completed the scales online. The research was approved by the Institutional Research Board of Bolu Abant Izzet Baysal University, Turkey.

\subsubsection{American sample}

A total of 214 adults who met the criteria completed an online study ( $46.73 \%$ women; 21 to 68 years old; Mage $=35.51$; $S D=10.06$ ) via Amazon's Mechanical Turk (MTurk). The participants received \$1.60 upon completion. The research was approved by the Institutional Research Board of University of North Carolina at Charlotte, USA.

\subsection{Measures}

\subsubsection{Expanded version of the PTG and PTD Inventory (PTGDI-X)}

PTG and PTD were assessed using the Posttraumatic Growth and Posttraumatic Depreciation Inventory - Expanded version (PTGDI-X) and the translated version for each country. The PTGDI-X is a 50 -item inventory that measures the aforementioned five domains of PTG (PTGI-X: Tedeschi et al., 2017) and PTD. Participants indicated the degree to which they did or did not experience a particular change from 0 to 5 , with separate scores calculated for PTG and PTD. Higher scores indicate greater growth or depreciation. The $\alpha$ for the total PTG was 0.93 (Italy) or higher, and also 0.93 (Turkey and Portugal) or higher for PTD.

\subsubsection{PTSD symptoms}

PTSD symptoms were assessed by the PTSD Checklist for DSM-5 (PCL-5; Blevins et al., 2015; Weathers et al., 2013) and its translated versions. The PCL-5 consists of 20 items. Participants indicated how much they have been bothered by each symptom using a 5-point scale from 0 to 4. Cronbach's $\alpha$ was 0.93 (Nepal) or above.

\subsubsection{Event centrality}

Event centrality was assessed by using the Centrality of Event Scale (CES: Berntsen \& Rubin, 2006) and its translated versions. The CES consists of 7 items. Participants indicated the degree to which the focal event was central to their identity using a 5-point scale from 1 to 5 . Cronbach's $\alpha$ ranged from 0.85 (Nepal) to 0.92 (Peru).

\subsubsection{Core beliefs examination}

Examination of core beliefs was assessed using the Core Beliefs Inventory (CBI: Cann, Calhoun, Tedeschi, Kilmer, et al., 2010) and its translated versions. The CBI consists of 9 items. Participants indicated the degree to which the focal event led them to seriously examine their core beliefs using a 6-point scale from 0 to 5. Cronbach's $\alpha$ ranged from 0.74 (Nepal) to 0.89 (Peru).

\subsubsection{Intrusive and deliberate rumination}

Intrusive and deliberate rumination in the aftermath of the trauma were assessed using the Event-Related Rumination Inventory (ERRI: Cann et al., 2011) and its translated versions. Participants responded to 10 items measuring intrusive rumination and 10 items measuring deliberate rumination using a 4-point scale from 0 to 3. Cronbach's $\alpha$ for the intrusive rumination was 0.94 or above, and for the deliberate rumination ranged from 0.88 (Italy and Portugal) to 0.92 (Japan). 


\subsubsection{Positive and negative disclosure}

An adapted version of the Cognitive and Emotional Processing from Disclosure Inventory (Müller et al., 2000) was used to assess positive aspects of disclosure with 11 items and negative aspects of disclosure with 9 items. Participants responded using a 6-point scale from 0 to 5. Cronbach's $\alpha$ for both positive and negative aspects of disclosure were 0.91 or above for all countries.

\subsection{Data analysis}

After obtaining the descriptive statistics, multigroup confirmatory factor analyses (MGCFAs) were conducted to test for measurement invariance of the PTGDI-X. Missing data were handled by using personlevel mean imputation. Correlations were then obtained between PTG and PTD. Two regression models were examined to assess the degree to which core beliefs examination, event centrality, intrusive and deliberate rumination, and positive/negative disclosure, in addition to demographics of gender and age, predicted PTG and PTD. Analyses were performed using IBM SPSS and AMOS software version 26.

\section{Results}

\subsection{MGCFAs of PTGDI-X}

Results of the MGCFAs are presented in Supplemental Table S2. Four models were tested and compared. Model 1 was baseline with no restrictions. Model 2 constrained the path coefficients from the PTG and PTD five factors to the observed variables (scale items), that is, factor loadings, to be equal across the ten countries. Model 3 constrained the path coefficients from PTG and PTD five factors to each higher order factor, PTG and PTD, to be equal, in addition to Model 2. Finally, Model 4 constrained the covariance between PTG and PTD to be equal, in addition to Model 3. Although the fit indices such as NFI and CFI were all poor, RMSEA showed the excellent fit. The difference of CFI between the models is less than 0.01 , indicating the factorial invariance model was satisfactory (Cheung \& Rensvold, 2002). Based on the fit indices such as AIC, factorial invariance with the higher order constructs (five factors to their PTG/PTD), that is Model 3, and the covariance between PTG and PTD, that is Model 4, were not supported. Therefore, Model 2 is considered as best fit to the data, meaning each of the PTG/PTD five factors was assessing the same constructs in the same way across the countries, however, the relationships between PTG and PTD as well as what constitutes PTG and PTD varied across the cultures. In the Model 2, Standardized regression weights were all greater than 0.77 for PTG and PTD. The item level factor loadings were all greater than 0.45 for both PTG and PTD. The correlation between PTG and PTD ranged from -0.37 to 0.23 .

\subsection{Descriptive statistics for PTGDI-X}

Descriptive statistics and the correlations among PTG, PTD, and PTSD symptoms for each country are presented in Supplemental Tables S3 and S4. Participants reported PTG more than PTD across all countries, ranging from $t(116)=4.27, p<.001$ for Italy to $t(195)=22.97$, $p<.001$ for Nepal. Correlations between PTG and PTD varied. Data from five countries showed no correlation, whereas the rest five countries indicated a curvilinear relationship.

\subsection{Different patterns of the predictors for PTG and PTD}

Two regression models were tested to examine the hypothesized relationships with a total sample (Supplemental Table S5). However, based on the results from MGCFAs, the same models were tested in each country, separately (Supplemental Tables S6 and S7). The positive impact of positive disclosure on PTG was consistent across the countries, however, the impact of the other predictors varied across the countries. Similarly, the negative impact of negative disclosure on PTD was consistent across the countries, however, the impact of the other predictors varied across the countries.

\section{Discussion}

This was the first study that analyzed international data to examine the nature of the relationships between PTG and PTD. The respondents overall reported more PTG than PTD, being consistent with previous studies (Cann, Calhoun, Tedeschi, Kilmer, et al., 2010; Michélsen et al., 2017). Results supported the weak invariance of factor loadings of the PTGDI-X across the countries, however, invariance in the relationships between the five PTG and PTD factors and their respective higher order factors as well as the covariance between PTG and PTD could not be assumed. In fact, of 10 countries, 5 (Australia, Japan, Turkey, Portugal, and Peru) showed no systematic relationships between PTG and PTD, whereas the other 5 countries (i.e., Italy, Nepal, the US, Germany, and Poland) indicated a curvilinear relationship, that is, PTG was more likely to be reported when a moderate level of PTD was also present. These results demonstrate that PTG and PTD are not on opposite ends of a single dimension across the board but rather co-exist, being consistent with findings from other studies (Kunz et al., 2019; Zięba et al., 2019). Given that PTD and PTSD symptoms were positively correlated with each other, in line with previous research (Barrington \& ShakespeareFinch, 2013), PTD was an appropriate indicator measuring the "negative" aspects of changes after a trauma. It should be noted, however, that some of the model fit indices such as TLI and CFI were poor, suggesting that there may be an alternative model that would fit better for each country. One study indicated that PTG and PTD may not reflect perceptions of positive and negative psychological changes at least in a Japanese sample (Oshiro et al., 2019). Future study should examine the paradoxical nature of trauma, instead of relying on the dichotomized way, such as either positive or negative outcomes alone.

The current study also revealed different patterns of the predictors for PTG and PTD in each country. Unlike our hypotheses, two main predictors, reexamination of core beliefs and deliberate rumination, showed a positive relationship with PTG only in some countries. Future studies should identify the reasons why reexamination of core beliefs was not a strong predictor for PTG in some samples (e.g., Nepal, US) as well as deliberate rumination (e.g., Nepal, Turkey). Similarly, the impact of intrusive rumination on PTG also varied. Intrusive rumination could be an indicator of psychological struggle, and thus it could be positively related to PTG (Cann, Calhoun, Tedeschi, Kilmer, et al., 2010). It could, however, also overlap the PTSD symptoms. Our hypotheses for PTD were only suppored in some countries. Intrusive rumination and centrality of the event did not consistently show the positive relationships with PTD. Similar to a previous study (Allbaugh et al., 2016), we expected event centrality to play a positive role in both PTG and PTD, however, it was only supported with a German sample.

One robust finding in the current study is the role of positive disclosure on PTG and negative disclosure on PTD. A contrasting relationship between positive disclosure and PTG and the relationship between the negative disclosure and PTD is consistent with the previous findings (Kroemeke et al., 2017). Regardless of the sampling characteristics, the experience of positive disclosure led to PTG in all countries studied. These findings suggest that the individual experience of PTG may be inseparable from social connections and is likely to be fostered when the person felt relieved and helped after they talked about the event. This type of positive disclosure did not, however, impede the PTD experience. On the other hand, negative experience of self-disclosure predicted PTD, except for Nepal, which may be due to the extremely low level of PTD reported in Nepal. When people reflected on their disclosure and felt worse or more confused, it led to PTD experience. Interestingly, negative disclosure did not preclude PTG experience across cultures. These findings indicate that the PTG and PTD processes are again not the opposite, and explained by a uniquet 
set of cognitive and social factors. Lack of positive predictors does not automatically cause negative changes. Similarly, lack of negative predictors does not seem to be enough to lead to PTG.

There are several limitations to be taken into account. For any findings derived from the cross-cultural data to be valid, it is important to establish methodological and sampling equivalence among the cultures. It is, however, highly challenging because the impact of culture often confounds with demographics. For example, Supplemental Table S4 presented cross-national differences in the PTG and PTD domains. However, we cannot know if the national background caused these differences. Studies have suggested that PTG and PTD may be affected by the types and combinations of trauma exposure (Michélsen et al., 2017). It would be ideal if we could test our hypotheses by looking at each event separately. However, the nature of the trauma people experienced is also confounded with their national background, geographic location, and social stigma when reporting the events. Due to this intricate relationship between trauma, demographics, and socialpsychological factors, the data would become unrepresentative if researchers selected a part of the data so these factors (e.g., type of the trauma) will be equivalent across cultures. We therefore used the data that were obtained from each country by setting a single criterion, that is, the data were included only when a respondent confirmed that their experience met the definition of trauma provided. Findings that were reported in this study were thus all based on this self-reported trauma. In addition, research procedures such as pencil-and-paper surveys versus online surveys, open-ended questions versus using a list to identify the focal event, as well as college students versus community sample, might have affected the current results. Future studies should investigate how cross-cultural dimensions such as individualistic-collectivistic and independent-interdependent characteristics might affect the pathways of PTG and PTD, to develop a culturally sensitive intervention for trauma survivors. This is particulary important for cultures, such as Nepal (Kohrt \& Hruschka, 2010), where the meanings of trauma and distress differ from that of Western societies.

In summary, the current study identified universal aspects, such as equivalence of factor loadings of the PTGDI-X and the impact of positive/negative disclosure on PTG and PTD, as well as culture-specific aspects, including the relationships between PTG and PTD, and different patterns of cognitive predictors for PTG and PTD. These findings suggest that it is essential to use the PTGDI-X to understand both the positive and negative aspects of post-traumatic experiences, and raise a possibility that binary pathways (i.e., positive and negative) following trauma may vary around the globe.

\section{CRediT authorship contribution statement}

Kanako Taku:Formal analysis, Conceptualization, Methodology, Data curation, Writing - original draft, Writing - review \& editing.Richard G. Tedeschi:Conceptualization, Methodology, Data curation, Writing - original draft, Writing - review \& editing.Jane Shakespeare-Finch:Conceptualization, Methodology, Data curation, Writing - original draft, Writing - review \& editing.Daniel Krosch:Conceptualization, Methodology, Data curation, Writing original draft, Writing - review \& editing.Georgina David:Conceptualization, Methodology, Data curation, Writing original draft, Writing - review \& editing.Doris Kehl: Conceptualization, Methodology, Data curation, Writing - original draft, Writing - review \& editing.Selina Grunwald:Formal analysis, Conceptualization, Methodology, Data curation, Writing - original draft, Writing - review \& editing.Annunziata Romeo: Conceptualization, Methodology, Data curation, Writing - original draft, Writing - review \& editing.Marialaura Di Tella: Conceptualization, Methodology, Data curation, Writing - original draft, Writing - review \& editing.Kiyoko Kamibeppu: Conceptualization, Methodology, Data curation, Writing - original draft, Writing - review \& editing.Takafumi Soejima:
Conceptualization, Methodology, Data curation, Writing - original draft, Writing - review \& editing.Kohichi Hiraki:Conceptualization, Methodology, Data curation, Writing - original draft, Writing - review \& editing.Rebekah Volgin:Conceptualization, Methodology, Data curation, Writing - original draft, Writing - review \& editing.Sandesh Dhakal:Conceptualization, Methodology, Data curation, Writing - original draft, Writing - review \& editing.Mariusz Zięba:Conceptualization, Methodology, Data curation, Writing original draft, Writing - review \& editing.Catarina Ramos: Conceptualization, Methodology, Data curation, Writing - original draft, Writing - review \& editing.Romina Nunes:Conceptualization, Methodology, Data curation, Writing - original draft, Writing - review \& editing.Isabel Leal:Conceptualization, Methodology, Data curation, Writing - original draft, Writing - review \& editing.Patrícia Gouveia:Conceptualization, Methodology, Data curation, Writing original draft, Writing - review \& editing.Carolina C. Silva: Conceptualization, Methodology, Data curation, Writing - original draft, Writing - review \& editing.Pamela Núñez Del Prado Chaves:Conceptualization, Methodology, Data curation, Writing original draft, Writing - review \& editing.Claudia Zavala: Conceptualization, Methodology, Data curation, Writing - original draft, Writing - review \& editing.Andrea Paz:Conceptualization, Methodology, Data curation, Writing - original draft, Writing - review \& editing.Emre Senol-Durak:Conceptualization, Methodology, Data curation, Writing - original draft, Writing - review \& editing.Atsushi Oshio:Formal analysis.Amy Canevello:Conceptualization, Methodology, Data curation, Writing - original draft, Writing - review \& editing.Arnie Cann:Conceptualization, Methodology, Data curation, Writing - original draft, Writing - review \& editing.Lawrence G. Calhoun:Conceptualization, Methodology, Data curation, Writing - original draft, Writing - review \& editing.

\section{Appendix A. Supplementary data}

Supplementary data to this article can be found online at https:// doi.org/10.1016/j.paid.2020.110222.

\section{References}

Allbaugh, L. J., Wright, M. O., \& Folger, S. F. (2016). The role of repetitive thought in determining posttraumatic growth and distress following interpersonal trauma. Anxiety, Stress, \& Coping, 29, 21-37. https://doi.org/10.1080/10615806.2015. 1015422.

American Psychiatric Association (2000). Diagnostic and statistical manual of mental disorders: DSM-IV-TR. Washington, DC: American Psychiatric Association.

Baker, J. M., Kelly, C., Calhoun, L. G., Cann, A., \& Tedeschi, R. G. (2008). An examination of posttraumatic growth and posttraumatic depreciation: Two exploratory studies. Journal of Loss and Trauma, 13, 450-465. https://doi.org/10.1080/ 15325020802171367.

Barrington, A., \& Shakespeare-Finch, J. (2013). Posttraumatic growth and posttraumatic depreciation as predictors of psychological adjustment. Journal of Loss and Trauma, 18, 429-443. https://doi.org/10.1080/15325024.2012.714210.

Berntsen, D., \& Rubin, D. C. (2006). The centrality of event scale: A measure of integrating a trauma into one's identity and its relation to post-traumatic stress disorder symptoms. Behaviour Research and Therapy, 44, 219-231. https://doi.org/10.1016/j. brat.2005.01.009.

Blevins, C. A., Weathers, F. W., Davis, M. T., Witte, T. K., \& Domino, J. L. (2015). The posttraumatic stress disorder checklist for DSM-5 (PCL-5): Development and initial psychometric evaluation. Journal of Traumatic Stress, 28, 489-498. https://doi.org/ $10.1002 /$ jts. 22059.

Cann, A., Calhoun, L. G., Tedeschi, R. G., Kilmer, R. P., Gil-Rivas, V., Vishnevsky, T., \& Danhauer, S. C. (2010). The core beliefs inventory: A brief measure of disruption in the assumptive world. Anxiety, Stress, \& Coping, 23, 19-34. https://doi.org/10.1080/ 10615800802573013.

Cann, A., Calhoun, L. G., Tedeschi, R. G., \& Solomon, D. T. (2010). Posttraumatic growth and depreciation as independent experiences and predictors of well-being. Journal of Loss and Trauma, 15, 151-166. https://doi.org/10.1080/15325020903375826.

Cann, A., Calhoun, L. G., Tedeschi, R. G., Triplett, K. N., Vishnevsky, T., \& Lindstrom, C. M. (2011). Assessing posttraumatic cognitive processes: The event related rumination inventory. Anxiety, Stress, \& Coping, 24, 137-156. https://doi.org/10.1080/ 10615806.2010 .529901$.

Cheung, G. W., \& Rensvold, R. B. (2002). Evaluating goodness of fit indexes for testing 
measurement invariance. Structural Equation Modeling: A Multidisciplinary Journal, 9, 233-255. https://doi.org/10.1207/S15328007SEM0902 5.

Groleau, J. M., Calhoun, L. G., Cann, A., \& Tedeschi, R. G. (2013). The role of centrality of events in posttraumatic distress and posttraumatic growth. Psychological Trauma: Theory, Research, Practice, and Policy, 5, 477-483. https://doi.org/10.1037/ a0028809.

Gul, E., \& Karanci, A. N. (2017). What determines posttraumatic stress and growth following various traumatic events? A study in a Turkish community sample. Journal of Traumatic Stress, 30, 54-62. https://doi.org/10.1002/jts.22161.

Kohrt, B. A., \& Hruschka, D. J. (2010). Nepali concepts of psychological trauma: The role of idioms of distress, ethnopsychology and ethnophysiology in alleviating suffering and preventing stigma. Culture, Medicine, and Psychiatry, 34, 322-352. https://doi. org/10.1007/s11013-010-9170-2.

Kroemeke, Bargiel-Matusiewicz, \& Kalamarz (2017). Mixed psychological changes following mastectomy: Unique predictors and heterogeneity of posto-traumatic growth and post-traumatic depreciation. Frontiers in Psychology, 8, 1245. https://doi.org/10. 3389/fpsyg.2017.01245.

Kunz, S., Fellinghauer, C., \& Peter, C. (2019). Measuring posttraumatic growth and depreciation after spinal cord injury: A Rasch analysis. Rehabilitation Psychology, 64, 407-424. https://doi.org/10.1037/rep0000288.

Michélsen, H., Therup-Svedenlöf, C., Backheden, M., \& Schulman, A. (2017). Posttraumatic growth and depreciation six years after the 2004 tsunami. European Journal of Psychotraumatology, 8, Article 1302691. https://doi.org/10.1080/ 20008198.2017.1302691.

Mosher, C. E., Adams, R. N., Helft, P. R., O'Neil, B. H., Shahda, S., Rattray, N. A., \& Champion, V. L. (2017). Positive changes among patients with advanced colorectral cancer and their family caregivers: A qualitative analysis. Psychology \& Health, 32, 94-109. https://doi.org/10.1080/08870446.2016.1247839.

Müller, J., Beauducel, A., Raschka, J., \& Maercker, A. (2000). Kommunikationsverhalten nach politischer Haft in der DDR - Entwicklung eines Fragebogens zum Offenlegen der Traumaerfahrungen [Communication after political imprisonment: disclosure of the traumatic experiences]. Zeitschrift fuer Politische Psychologie. Vol. 8. Zeitschrift fuer Politische Psychologie (pp. 413-427).

Nordstrand, A. E., Hjemdal, O., Holen, A., Reichelt, J. G., \& Bøe, H. J. (2017). Measuring psychological change after trauma: Psychometric properties of a new bi-directional scale. Psychological Trauma: Theory, Research, Practice, and Policy, 9, 696-705. https://doi.org/10.1037/tra0000270.

Oshiro, R., Kopitz, J., Soejima, T., Kibi, S., Kamibeppu, K., Sakamoto, S., \& Taku, K.
(2019). Perceptions of positive and negative changes for posttraumatic growth and depreciation: Judgments from Japanese undergraduates. Personality and Individual Differences, 137, 17-21. https://doi.org/10.1016/j.paid.2018.07.040.

Shakespeare-Finch, J., \& Lurie-Beck, J. (2014). A meta-analytic clarification of the relationship between posttraumatic growth and symptoms of posttraumatic distress disorder. Journal of Anxiety Disorder, 28, 223-229. https://doi.org/10.1016/j.janxdis. 2013.10.005.

Shakespeare-Finch, J., Martinek, E., Tedeschi, R. G., \& Calhoun, L. G. (2013). A qualitative approach to assessing the validity of the posttraumatic growth inventory. Journal of Loss and Trauma, 18, 572-591. https://doi.org/10.1080/15325024.2012. 734207.

Slavin-Spenny, O. M., Cohen, J. L., Oberleitner, L. M., \& Lumley, M. A. (2011). The effects of different methods of emotional disclosure: Differentiating post-traumatic growth from stress symptoms. Journal of Clinical Psychology, 67, 993-1007. https://doi.org/ 10.1002/jclp. 20750.

Taku, K., Cann, A., Calhoun, L. G., \& Tedeschi, R. G. (2008). The factor structure of the posttraumatic growth inventory: A comparison of five models using confirmatory factor analysis. Journal of Traumatic Stress, 21, 158-164. https://doi.org/10.1002/jts. 20305.

Tedeschi, R. G., Can, A., Taku, K., Senol-Durak, K., \& Calhoun, L. G. (2017). The posttraumatic growth inventory: A revision integrating existential and spiritual change. Journal of Traumatic Stress, 30, 11-18. https://doi.org/10.1002/jts.22155.

Tedeschi, R. G., Shakespeare-Finch, J., Taku, K., \& Calhoun, L. G. (2018). Posttraumatic growth: Theory, research, and applications. NY: Routledge.

Tedeshci, R. G., \& Blevins, C. L. (2015). From mindfulness to meaning: Implications for the theory of posttraumatic growth. Psychological Inquiry, 26, 373-376. https://doi. org/10.1080/1047840X.2015.1075354.

Weathers, F. W., Litz, B. T., Keane, T. M., Palmieri, P. A., Marx, B. P., \& Schnurr, P. P. (2013). The PTSD checklist for DSM-5 (PCL-5). Retrieved from www.ptsd.va.gov.

Weiss, T., \& Berger, R. (Eds.). (2010). Posttraumatic growth and culturally competent practice: Lessons learned from around the globe. Hoboken, NJ: Wiley.

Wilson, B., Morris, B. A., \& Chambers, S. (2014). A structural equation model of posttraumatic growth after prostate cancer. Psycho-Oncology, 23, 1212-1219. https://doi. org $/ 10.1002 /$ pon. 3546 .

Zięba, M., Wiecheć, K., Biegańska-Banaś, J., \& Mieleszczenko-Kowszewicz, W. (2019). Coexistence of post-traumatic growth and post-traumatic depreciation in the aftermath of trauma: Qualitative and quantitative narrative analysis. Frontiers in Psychology, 10, 687. https://doi.org/10.3389/fpsyg.2019.00687. 